\title{
Fitting hydrodynamical simulations to broad-band GRB afterglow data
}

\section{F. Knust*}

Max Planck Institute for extraterrestrial physics, 85748 Garching, Germany

E-mail: fknustampe.mpg.de

\section{H.J. Van Eerten}

Max Planck Institute for extraterrestrial physics, 85748 Garching, Germany

E-mail: hveerten@mpe.mpg.de

\section{J. Greiner}

Max Planck Institute for extraterrestrial physics, 85748 Garching, Germany

E-mail: jcg@mpe.mpg.de

\section{R. Filgas}

IEAP, Czech Technical University in Prague, 12800 Praha 2, Czech Republic

E-mail: robert.filgas@utef.cvut.cz

The Gamma-Ray burst Optical Near-infrared Detector (GROND) offers early time data of Gamma Ray Burst (GRB) afterglows in 4 optical and 3 near-infrared (NIR) bands simultaneously. We extended the SCALEFIT package to allow for joint fits of GROND and Swift/XRT data. Using GRB 140512A, we demonstrate the improvements over fitting X-ray data only. We are able to constrain some of the parameters of the fireball model, for others we derive limits.

Swift: 10 Years of Discovery,

2-5 December 2014

La Sapienza University, Rome, Italy

\footnotetext{
* Speaker.
} 


\section{Fitting based on hydrodynamical simulations}

We report on our project to extended the SCALEFIT package (Ryan et al. 2015, van Eerten et al. in preparation) to allow for joint fits of GRB afterglow data from GROND [6] and Swift/XRT [1]. For the first time we apply it to broad-band data, using GRB 140512A as an example. We cover different spectral regimes and thus are able to break some degeneracies, that occur when applying SCALEFIT to Swift/XRT data only [9].

The fireball model describes GRB afterglows as synchrotron emission. We model the whole time evolution of the Spectral Energy Distribution (SED) of the afterglow, which has the advantage that we can use data from every spectral regime at every point in time. There is no need for synchronous data gathering from different instruments (or interpolations or extrapolations), as would be the case for single SED fitting. The afterglow model is based directly on hydrodynamical simulations $[13,14]$ that cover the jet dynamics in more detail than the standard asymptotic analytical approaches [10], opening the new possibility to constrain the jet opening angle $\theta_{0}$ and the observer angle $\theta_{\text {obs }}$.

Full details of SCALEFIT can be found in [9]. In short: These simulations cover a range of opening angles $\theta_{0}$ and can be re-scaled between isotropic equivalent explosion energy $E_{0}$ and circumburst medium density $n_{0}$ [15]. A simulation run takes days to weeks and the results of those simulations are stored in templates. Based on these, a radiative transfer code calculates the emission characteristics under an observer angle $\theta_{o b s}$ with luminosity-distance $d_{L}$ (derived from redshift $z$ ) for a given set of micro-physical parameters (electron power law distribution slope $p$, fraction of energy in the magnetic field $\varepsilon_{B}$ and fraction of energy in electrons $\varepsilon_{e}$ ). The crucial part for simulation based fitting is to pre-calculate the whole process for a given set of parameters $\Theta=\left\{E_{0}, n_{0}, \theta_{0}, \theta_{o b s}, p, \varepsilon_{e}, \varepsilon_{B}, d_{L}, z\right\}$ and compress the data in a way that the results are accessible from every iteration step of the fitting process. The results are stored in tables of the time evolution for characteristic quantities for the afterglow SED: cooling frequency $v_{c}$, peak frequency $v_{m}$ and peak flux $f_{p}$. Since the model assumes synchrotron radiation as the emission process, the SED is just a series of connected power-laws, separated by the characteristic frequencies $v_{m}$ and $v_{c}$, with slopes following the relations from [10].

The SCALEFIT package uses parallel tempered Markov Chains to minimize the $\chi^{2}$ [5]. This Markov Chain Monte Carlo (MCMC) method is capable of completely exploring a high dimensional parameter space on a justifiable time scale, making the fitting process very robust towards degeneracies between parameters and multi-modal behavior of the Posterior Distribution Function (PDF).

\section{GRB 140512A, an example}

On 12.05.2014 at 19:21UT, the Burst Alert Monitor (BAT) triggered on GRB140512A and the XRT began observing 98.4 seconds thereafter [8]. GROND began its follow up observations on 13.05.2014 at 03:36UT, around $8 \mathrm{hrs}$ after the trigger, for a total of 58 observations, distributed over 4 nights. To reduce the GROND data we used our IRAF/PyRAF [12] based pipeline [16, 7]. GROND magnitudes are corrected for galactic foreground extinction with an $A_{V}=0.4$ mag towards the direction of the burst [11], and using the CCM law with $R_{V}=3.08$. For this individual GRB the GROND SED does not indicate significant host extinction nor host flux contribution. The XRT 
light curve (integrated over the $0.3-10 \mathrm{keV}$ range) is fetched from the Swift online repository [3] and corrected for absorption. Unlike [9] we use broadband data, covering several orders of magnitude in energy, which raises the issue that a fit always will be biased by the spectral regime with the most data points. To mitigate this effect, we added a weighing scheme which ensures that both instruments contribute equally to the fit. We use a redshift of $z=0.725$ [2] leading to a luminosity distance $d_{L}=1.37 \times 10^{28} \mathrm{~cm}$ with standard cosmology $\left(H_{0}=71 \frac{\mathrm{km} / \mathrm{s}}{\mathrm{Mpc}}, \Omega_{m}=0.27, \Omega_{\Lambda}=0.73\right)$. Since the model describes the deceleration phase of the GRB, we omit the data before $t_{o b s}=10000$ $\mathrm{s}$, and run the fitting process.

The light curve (data and best fitting model) are shown in Fig. 1, a visualization of the complete PDF for our broad band data set is shown in Fig. 2. Compared to a fit with XRT data only (see Fig.3), the distributions are substantially more narrow.

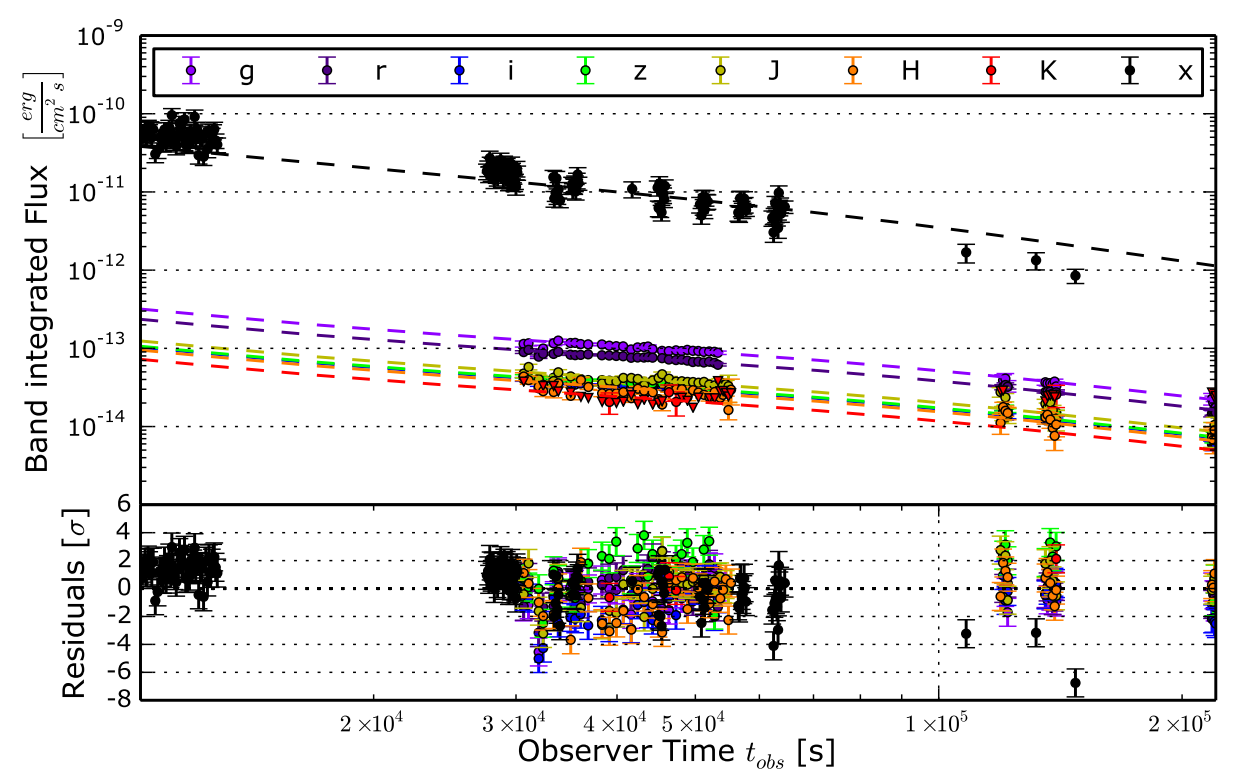

Figure 1: Light curve of GRB140512A. The dots are data points and their $1 \sigma$ error. The dashed lines are the best fitting model. (Preliminary)

\section{Discussion}

In general, the fit captures the optical/NIR temporal curvature. An offset of 1 to $2 \sigma$ to the model can be seen, especially in the optical bands. This may be due to extinction in the host, which we did not account for, or due to small-scale effects not covered in the dust maps for the galactic extinction. In the X-ray regime, the last 3 data points are not well modelled. While from a statistical point of view 3 out of 146 data points can be off the model for a $68 \%$ confidence level, having the 3 data points next to each other indicates that the fitting process missed to model a break in the light curve.

The chromaticity of the break rules out a clean jet break. Additionally, the transition from an X-ray temporal power law slope $\alpha$ of $1.25_{-0.06}^{+0.06}$ before the break to $1.94_{-0.17}^{+0.22}$ after the break at $t=5.25_{-1.19}^{+0.86} \times 10^{4} \mathrm{~s}$ (from the live XRT GRB catalogue ${ }^{1}$ ) does not fulfill the relations from

\footnotetext{
${ }^{1}$ http://www.swift.ac.uk/xrt_live_cat/598819
} 


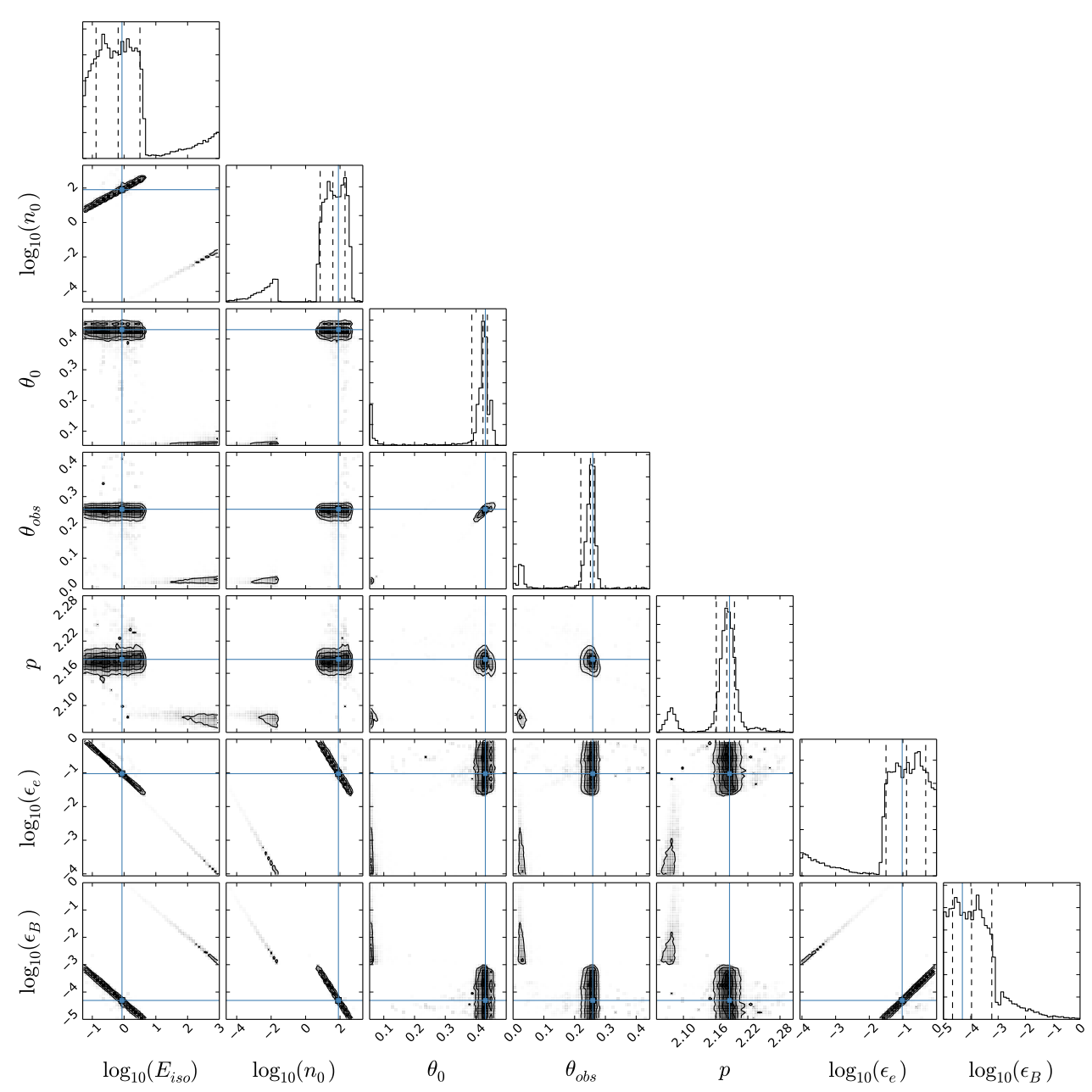

Figure 2: PDF of the MCMC sample for GROND and XRT data for GRB 140512A. The contour plots show the correlations between all pairs of parameters. The histograms in the diagonal show the marginalized probabilities for each parameter. The dotted vertical lines are the median and its $68 \%$ confidence range. The blue dot and lines mark the best-fit. $E_{i s o}$ is given in units of $10^{53} \mathrm{erg}, n_{0}$ is given in $\mathrm{cm}^{-3}, \theta_{0}$ and $\theta_{o b s}$ are given in radians. All other quantities are dimensionless. (Preliminary)

[10] for the pre- and post cooling break $\alpha$ for any electron distribution index $p$, neither for an Inter Stellar Medium (ISM) type environment nor for a stellar-wind type environment. Since the more complex dynamics of the simulations (assuming ISM) also fails to model the break, it becomes difficult to reconcile the X-ray break with a single transition of a basic forward-shock synchrotron model.

As an alternative explanation, a temporal variation of $\varepsilon_{B}$ as suggested by [4] is also unlikely, since with $F_{v} \propto \varepsilon_{B}^{(p-2) / 4}$ (above the cooling break, in the ISM case) the effect would be too small to explain the steepening of the light curve.

We are able to constrain $\theta_{0}=0.42_{-0.04}^{+0.01} \mathrm{rad}=24.1_{-2.3}^{+0.6} \mathrm{deg}, \theta_{\text {obs }}=0.25_{-0.03}^{+0.01} \mathrm{rad}=14.3_{-2.3}^{+0.6}$ deg and $p=2.18_{-0.02}^{+0.01}$. For the other micro-physical parameters we derive limits $10^{-2} \lesssim \varepsilon_{e}<1$ and $\varepsilon_{B} \lesssim 10^{-3}$ (all numbers are still under consideration). Pairwise degeneracies between e.g. $E_{i s o}$ and $n_{0}$ can only be reduced by including radio or sub-mm data, which would constrain the peak 


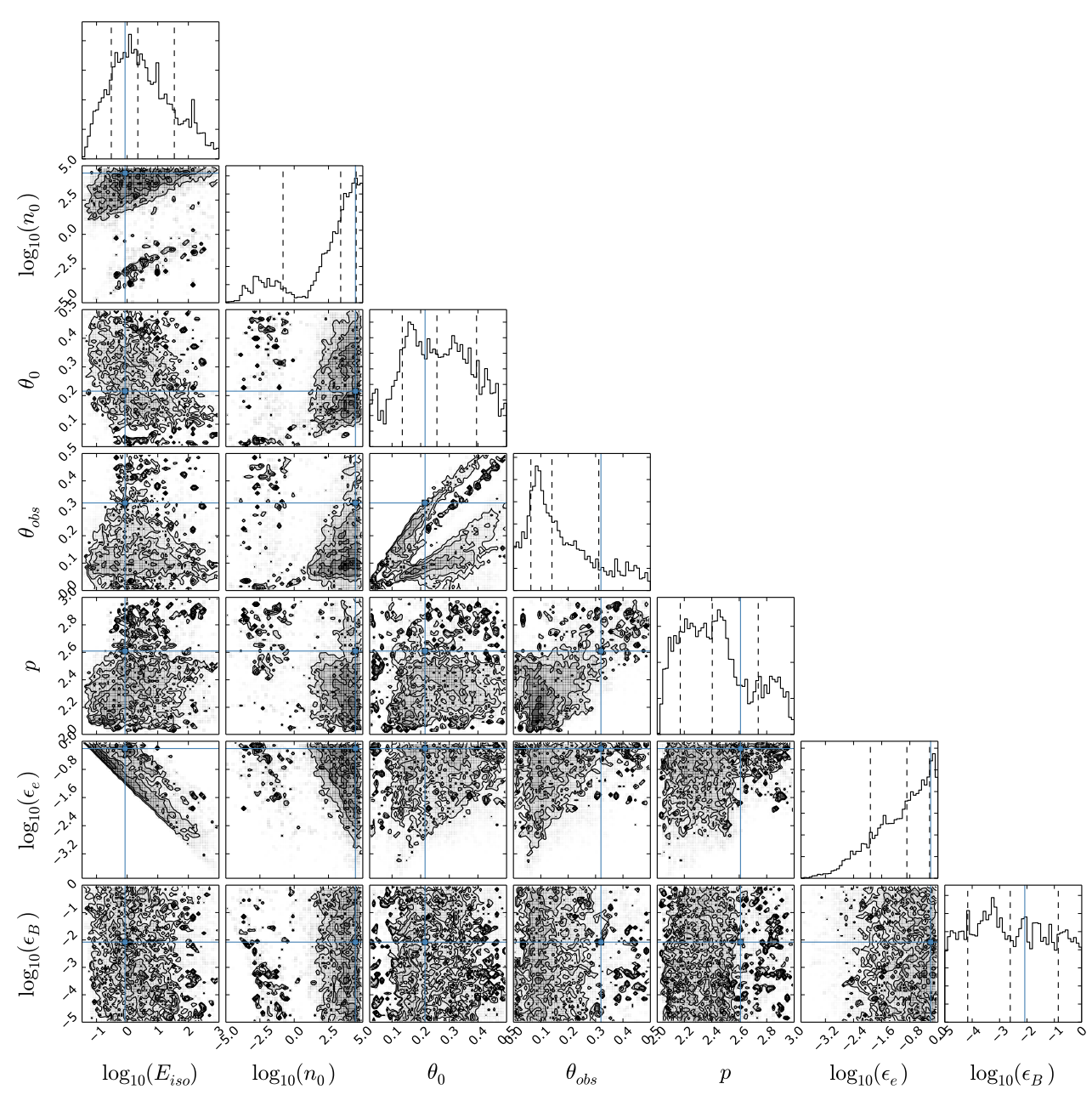

Figure 3: PDF for the same burst, but with XRT data only. In comparison to broad band data, the fitting parameters are barely constrained. (Preliminary)

frequency $v_{m}$ of the synchrotron spectrum.

\section{Summary and outlook}

We have demonstrated the feasibility of fitting hydrodynamical simulations to non-simultaneous broad band data with 405 individual data points. These simulations treat the jet dynamics in substantially more detail than asymptotic analytical approaches, opening a new possibility to constrain the observer angle. With combined optical/NIR and X-ray data, the parameters of interest are much better constrained, in comparison to the fit obtained by using data from a single spectral regime. Since we model the time evolution of the SED, observations in different spectral regimes do not necessarily have to be time synchronous. The applied statistical method is capable of exploring a high dimensional parameter space effectively, and is robust towards multi-modal behavior of the parameter distribution.

For the future we will apply this kind of analysis to the full GROND sample. The GROND instrument has observed basically every GRB visible from the ESO La Silla observatory for the 
last 7 years, building up a sample of over 200 afterglow detections, around half of them with a data coverage sufficient for light curve fitting. The implementation of data from additional spectral regimes will be a logical next step.

\section{Acknowledgments}

We acknowledge the contributions of all GROND observers, especially K. Varela, C. Delvaux, J. Graham, S.Schmidl, M. Tanga. JG acknowledges support by the DFG cluster of excellence "Origin and Structure of the Universe" (www.universe-cluster.de). HJvE is supported by the Alexander von Humboldt foundation. RF acknowledges support through the German Academic Exchange Service DAAD PPP-57066218 and through the Czech Ministry of Education, Youth and Sports, Grant 7AMB14DE001. Part of the funding for GROND (both hardware as well as personnel) was generously granted from the Leibniz-Prize to Prof. G. Hasinger (DFG grant HA 1850/28-1). We acknowledge the support by the DFG Cluster of Excellence "Origin and Structure of the Universe". The simulations have been carried out in part on the computing facilities of the Computational Center for Particle and Astrophysics (C2PAP). This work made use of data supplied by the UK Swift Science Data Centre at the University of Leicester.

\section{References}

[1] D. N. Burrows et al., The Swift X-Ray Telescope, Space science reviews 120 (2005) 165-195

[2] A. de Ugarte Postigo et al., GRB 140512A: Redshift from NOT, GCN 16310 (2014)

[3] P. A. Evans et al., Methods and results of an automatic analysis of a complete sample of Swift-XRT observations of GRBs, MNRAS 397 (2009) 1177-1201

[4] R. Filgas et al., GRB 091127: The cooling break race on magnetic fuel, A\&A 535 (2011) A57

[5] D. Foreman-Mackey et al., emcee: The MCMC Hammer, ASP 125 (2013) 306-312

[6] J. Greiner et al., GROND, a 7-Channel Imager, ASP 120 (2008) 405-424

[7] T. Krühler et al., The $2175 \AA$ A Dust Feature in a Gamma-Ray Burst Afterglow at Redshift 2.45, ApJ 685 (2008) 376-383

[8] C. Pagani et al., GRB 140512A: Swift detection of a burst with an optical counterpart, GCN 16249 (2014)

[9] G. Ryan et al., Gamma-Ray Bursts are Observed Off-axis, ApJ 799 (2015) 3

[10] R. Sari et al., Spectra and Light Curves of GRB Afterglows, ApJ 497 (1998) L17

[11] E. F. Schlafly and D. P. Finkbeiner, Measuring Reddening with Sloan Digital Sky Survey Stellar Spectra and Recalibrating SFD, ApJ 737 (2011) 103

[12] D. Tody et al., IRAF in the Nineties, Astronomical Data Analysis Software and Systems II, ASP Conference Series 52 (1993) 173

[13] H. van Eerten et al., Off-axis Gamma-ray Burst Afterglow Modeling Based on a Twodimensional Axisymmetric Hydrodynamics Simulation, ApJ 722 (2010) 235-247

[14] H. J. van Eerten et al., Gamma-ray burst afterglows from transrelativistic blast wave simulations, MNRAS 403 (2010) 300-316

[15] H. J. van Eerten and A. I. MacFadyen. Gamma-Ray Burst Afterglow Scaling Relations for the Full Blast Wave Evolution, ApJ 747 (2012) L30

[16] A. K. Yoldaş et al., First Results of GROND, AIP Conf. Series 1000 (2008) 227-231 